Meta

Journal des traducteurs

Translators' Journal

\title{
Les problèmes linguistiques de la traduction automatique des prédicats nominaux entre l'arabe et le français
}

\section{Béchir Ouerhani}

Volume 53, numéro 2, juin 2008

La traduction des séquences figées

The Translation of Frozen Sequences

URI : https://id.erudit.org/iderudit/018526ar

DOI : https://doi.org/10.7202/018526ar

Aller au sommaire du numéro

Éditeur(s)

Les Presses de l'Université de Montréal

ISSN

0026-0452 (imprimé)

1492-1421 (numérique)

Découvrir la revue

Citer cet article

Ouerhani, B. (2008). Les problèmes linguistiques de la traduction automatique des prédicats nominaux entre l'arabe et le français. Meta, 53(2), 407-419.

https://doi.org/10.7202/018526ar
Résumé de l'article

Cet exposé part de l'idée que l'opération de traduction des constructions à prédicats nominaux est une mise en relation d'équivalence entre leurs schémas d'arguments respectifs en L1 et L2. Ceci revient à faire des descriptions linguistiques exhaustives et détaillées des éléments concernés. Ainsi, nous essayerons de montrer que l'élaboration des dictionnaires des équivalents - à partir d'une première grille proposée par Gaston Gross - doit nécessairement prendre en compte les spécificités des deux langues. Pour l'arabe par exemple, il est nécessaire d'inclure les informations relatives aux compatibilités entre un nom prédicatif et les verbes supports qu'il sélectionne, le degré d'appropriation de ceux-ci, ainsi que les propriétés morphologiques du prédicat et leur impact sur les reformulations par paraphrase. Ce dernier point relève d'une des spécificités de l'arabe, à savoir le fonctionnement du système de dérivation qui met en relation une multitude de formes à partir d'une seule matière consonantique.
Ce document est protégé par la loi sur le droit d'auteur. L’utilisation des services d’Érudit (y compris la reproduction) est assujettie à sa politique d'utilisation que vous pouvez consulter en ligne.

https://apropos.erudit.org/fr/usagers/politique-dutilisation/ 


\title{
Les problèmes linguistiques de la traduction automatique des prédicats nominaux entre l'arabe et le français
}

\author{
BÉCHIR OUERHANI \\ Université de Sousse, Sousse, Tunisie \\ bechir_ouerhani@yahoo.fr
}

\section{RÉSUMÉ}

Cet exposé part de l'idée que l'opération de traduction des constructions à prédicats nominaux est une mise en relation d'équivalence entre leurs schémas d'arguments respectifs en $\mathrm{L}_{1}$ et $\mathrm{L} 2$. Ceci revient à faire des descriptions linguistiques exhaustives et détaillées des éléments concernés. Ainsi, nous essayerons de montrer que l'élaboration des dictionnaires des équivalents - à partir d'une première grille proposée par Gaston Gross - doit nécessairement prendre en compte les spécificités des deux langues. Pour l'arabe par exemple, il est nécessaire d'inclure les informations relatives aux compatibilités entre un nom prédicatif et les verbes supports qu'il sélectionne, le degré d'appropriation de ceux-ci, ainsi que les propriétés morphologiques du prédicat et leur impact sur les reformulations par paraphrase. Ce dernier point relève d'une des spécificités de l'arabe, à savoir le fonctionnement du système de dérivation qui met en relation une multitude de formes à partir d'une seule matière consonantique.

\section{ABSTRACT}

This paper explores the idea that translating nominal predicate constructions means correlating equivalents of predicative structures consecutively in $L 1$ and $L 2$. This demands detailed and exhaustive linguistic descriptions of the elements in question. We will try to show that formulating dictionaries of equivalents - starting from a model suggested by Gaston Gross - must necessarily respect the specificities of both languages. Concerning Arabic, for instance, it's necessary to state information about the compatibility and aptness between a given nominal predicate and the support verbs it selects, as well as the impact of morphological properties on reformulation by paraphrases. This last point refers to the Arabic features, namely the derivation system that establishes a link between varied forms springing from one given root.

\section{MOTS-CLÉS/KEYWORDS}

traduction automatique, construction à verbe support, collocation, paraphrase, prédicat

\section{Introduction}

Ce travail s'inscrit dans le cadre théorique qui analyse la phrase en termes de prédicat et d'arguments (cf. Harris 1964; 1976; Gross 1981 et les travaux de G. Gross et autres élaborés au sein du Laboratoire de linguistique informatique ${ }^{1}$ ). Il part du fait que la traduction, automatique et autre, consiste à traduire des schémas d'arguments et non pas des unités lexicales et qu'il s'agit donc de «trouver» les emplois équivalents. Dans ce qui suit, nous nous intéresserons précisément à la description linguistique que nécessite la traduction automatique des noms prédicatifs (Npred.). 
Dans un premier temps, nous exposerons les cas de figure possibles lors du passage d'une langue source (L1) à une langue cible (L2) quant à la traduction des constructions à Npred. Dans un deuxième temps, nous nous arrêterons sur la méthode d'analyse. Dans une troisième partie, nous donnerons des échantillons de l'application de la démarche exposée.

\section{Le passage de $\mathrm{L} 1$ à $\mathrm{L} 2$ : les cas de figure possibles}

Rappelons que lorsque nous parlons de noms prédicatifs, il s'agit en fait de combinaisons Vsup._Npred., c'est-à-dire des «constructions à verbes supports». Les multiples travaux sur ces constructions, et spécialement sur leur traduction, ont mis l'accent sur «le caractère imprévisible» des Vsup. C'est-à-dire que lors du passage de L1 à L2, on ne peut pas prévoir quel Vsup. pour quel Npred. Ce qui nécessite, comme nous allons le voir plus loin, des listes d'équivalences préétablies entre les langues en question pour garantir un «bon» transfert.

Nous pouvons énumérer trois cas de figure quant à la traduction des constructions à Vsup. d'une manière générale ${ }^{2}$ :

\subsection{Vsup. + Npred. $\rightarrow$ Vsup. + Npred. (avec le même Vsup.)}

C'est le cas qui pose le moins de problèmes puisqu'il s'agit dans les deux langues de combinaisons Vsup_Npred.:

$$
\begin{aligned}
& \text { قام بجوْلة } \\
& \text { qa:ma bi zawla } \\
& \text { faire-prép.-promenade } \\
& \text { مارس الجر احة } \\
& \text { ma:rasa- 1- 3ira:hata } \\
& \text { طر سؤ الا } \\
& \text { taraћa su?a:lan } \\
& \text { ارتكب (خطأ + جريمة + مخالفة) }
\end{aligned}
$$

Faire une promenade

Pratiquer la chirurgie

Poser une question

Commettre (une faute + un crime + une violation/transgression)

Il suffit, dans ce cas de faire le lien entre les deux constructions, puisqu'il y a une correspondance entre la combinaison dans L1 et son équivalent en L2. L'idéal serait donc d'avoir des suites Vsup._Npred. avec des Npred. de la même classe sémantique et des Vsup. du même type (générique/approprié/variante lexicale ou aspectuelle, etc.). Ainsi, dans la série d'exemples ci-dessus, chaque élément de la suite est le transfert direct de son correspondant dans l'autre langue:

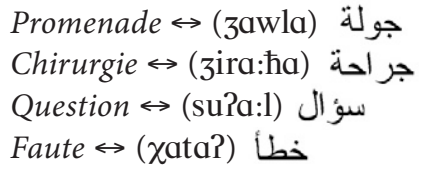

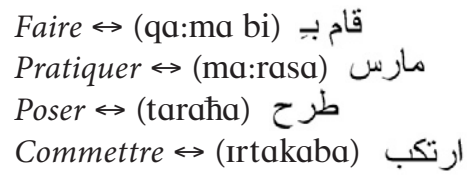


Crime $\leftrightarrow$ (3ai:ma) جريمة

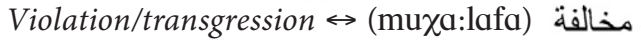

Le cas de figure ne couvre pas la totalité des emplois.

\subsection{Vsup. + Npred. $\rightarrow$ Vsup. + Npred. :}

Nous trouvons, en effet, un grand nombre de cas d' équivalence partielle» où nous avons, par exemple, un Npred. dans L1 qui sélectionne un Vsup. générique, et dont l'équivalent sélectionne un Vsup. approprié. Il est possible aussi d'avoir deux Vsup. appropriés mais de «types» différents. Examinons les exemples suivants:

$$
\begin{array}{r}
\text { رَفَعَ شَكوى } \\
\text { rafaca Jakwa: } \\
\text { lever-plainte }
\end{array}
$$

déposer une plainte

$$
\text { أسدى خدمة }
$$

Pasda: $\chi$ idmatan

rendre-service

offrir un service

Comme le montre la «traduction» littérale proposée, ces deux exemples illustrent bien le «caractère imprévisible» du choix du verbe support pour un Npred. donné. Même pour le cas d'équivalence, il faudra donc préciser le ou les verbes supports sélectionnés pour chaque classe de Npred., voire pour chaque Npred.

\subsection{Vsup. + Npred. $\rightarrow$ Vpred.}

Ce cas se caractérise par l'absence en L2 de construction à Vsup. correspondante à celle de L1. La solution est alors de fournir le verbe prédicatif équivalent. Nous positionnant toujours sous l'angle de la traduction automatique, nous avançons que le transfert ne peut se faire qu'une fois le travail linguistique bien mené à terme quant à l'élaboration des descriptions adéquates et pertinentes pour la traduction automatique.

Ceci implique la nécessité de l'élaboration de descriptions uniformes et exhaustives qui se basent sur un principe de parallélisme entre les langues concernées.

\section{Description uniformisée en vue de la traduction automatique et spécificités linguistiques}

Plusieurs auteurs (entre autres, Danlos 1994; Heylen et al. 1994; dans une autre optique: Namer et Schmidt 1993; Blanco et Buvet 1999; Ranchhod 2000) ont montré que la traduction automatique des constructions à Vsup passe par la correspondance entre les descriptions des Npred. dans chacune des langues concernées. D’une manière générale, ces études s'accordent sur le fait que la traduction de ce type de construction nécessite un traitement modulaire. C'est-à-dire l'élaboration d'un ensemble de programmes en mesure de garantir les trois étapes de la traduction: l'analyse, le transfert et la production (synthèse).

Pour illustrer les trois phases, nous reprenons ici, pour l'essentiel, le schéma donné par M. Alonso Ramos (1998: 42-43): 
1) Phase d'analyse:

- Identifier les constructions à Vsup et reconnaître le Npred.

- Constituer le schéma d’arguments déterminé par le Npred.

2) Phase de transfert:

- La transposition de la structure dans L2 selon les spécificités de la langue d'accueil.

3) Phase de production (ou synthèse):

- Chercher dans les dictionnaires de L2 les unités concernées par le schéma d’argument.

- Combiner le nouveau Npred. avec le Vsup. adéquat.

Il est évident que ces opérations ne sont possibles que si les listes descriptives des éléments en question sont établies, et que les informations linguistiques fournies sont systématiques et uniformes d'une langue à l'autre.

\subsection{La description linguistique uniformisée}

Il résulte de ce qui a précédé qu'il faut établir une grille uniforme qui serait en mesure d'intégrer systématiquement les informations nécessaires pour les opérations susmentionnées. La grille de description des Npred. doit contenir des informations du type:

- déterminer la classe sémantique du Npred., le nombre et la nature des arguments susceptibles d'être sélectionnés par le prédicat en question, ainsi que leur construction (les combinaisons syntaxiques possibles);

- décrire les différents déterminants du Npred;

- expliciter les différentes transformations qui le concernent (reformulations) telles que les constructions converses ${ }^{3}$, la formation du passif, la relation de paraphrase possible avec une autre forme (verbale ou adjectivale) du prédicat, si le Npred. est non autonome, etc.

Puisqu'il existe plusieurs cas de figure pour la traduction de ces constructions, il est indispensable de mentionner de façon explicite les possibilités de transfert pour chaque cas précis. Ce qui revient à dire qu'il faut énumérer les deux possibilités:

- construction à Vsup.: préciser dans ce cas s'il s'agit du même type de Vsup. ou non;

- verbe prédicatif.

Il est clair que cela n'est possible qu'une fois que la grille de description systématique et uniformisée sera élaborée dans les langues en question. Nous sommes amené donc à mettre l'accent sur la procédure de «listes d'équivalences » ou «classes d'équivalences» (Ranchhod-Marques 2000: 264) préétablies avec toutes les informations linguistiques nécessaires pour le bon transfert automatique d'un emploi de Npred. donné d'une langue L1 à une langue L2. Nous pourrions imaginer alors deux types de grilles de description en vue de la traduction: soit l'élaboration de grilles autonomes pour chacune des langues concernées, avec des liens systématiques sur les différents niveaux des modules conçus pour assurer le fonctionnement des trois étapes énumérées plus haut, soit la conception de grilles «multilingues» qui intègrent en parallèle les informations relatives à chacune des langues.

Nous préférons la première procédure, c'est-à-dire l'élaboration de grilles séparées. Nous exposerons les raisons de ce choix dans ce qui suit, notamment en 2. 2. 
Nous voudrions à ce propos commencer par fournir un échantillon de grille de description des classes sémantiques des Npred. Cette grille de description a été proposée par G. Gross dans divers articles et cours auxquels nous avons eu l'occasion d'assister. Elle concerne le français et l'allemand.

Dans un premier temps, nous avons pris la classe sémantique des «coups», dans l'état d'avancement où elle était, et nous avons essayé d'y insérer les informations concernant l'équivalent arabe, à savoir la classe de « darb». Voici le résultat:

$<$ coups, Schläge, darb $>$ :

gifle, claque, coup de pied, beigne

Ohrfeige, Schlag, Fusstritt

darba, saf $\varsigma a, \underline{t} a \varsigma n a$, latma

1- Nombre et nature des arguments:

$\mathrm{N}_{0}$ : hum $4 / \mathrm{N}_{1}$ : hum $/ \mathrm{N}_{2}$ : sur, (auf, in, ?ala:) $\mathrm{Npc}$

2- Verbes supports basiques:

donner/recevoir

geben/bekommen, kriegen

wazzaha, Pașta:

3- Déterminants

Quantifieurs

4- Verbes supports aspectuels:

Duratif

Inchoatif: esquisser

Intensif: asséner

Itératif: bourrer de,

Ponctuel

Progressif

Terminatif

5- Verbes supports appropriés:

Coller, flanquer, porter

Versetzen, verpassen

saddada

6- Verbes supports métaphoriques:

Administrer

verabreichen

3a:da bi

7- Restructurations:

$B$ a reçu une gifle de $A$

$B$ hat von A eine Ohrfeige bekommen

$B$ talaqqa: latmatan min A

8- Constructions causatives sur le verbe support

9- Prédicats verbaux appropriés

10- Prédicats adjectifs appropriés

11- Adverbiaux appropriés 
Il est possible donc, a priori, d'uniformiser la description selon ce qui a été proposé pour la langue arabe.

Nous pensons, cependant, qu'il est nécessaire de prendre en compte d'autres paramètres qui constituent les spécificités d'une langue aussi différente du français et de l'allemand sur plusieurs plans, notamment celui de la morphosyntaxe. Il suffit de penser à la quasi-systématicité du système dérivationnel pour comprendre qu'il faut tout de suite prendre en compte ces spécificités. En effet, il est pertinent pour les classes d'équivalence de préciser en arabe les différentes paraphrases possibles construites sur le verbe morphologiquement apparenté au Npred, ainsi que la possibilité ou non de la construction du passif, etc.

\subsection{Spécificités de l'arabe}

Il s'agit pour l'essentiel de faits relevant de la systématicité des faits morphologiques en arabe, dont l'impact est évident sur les grilles de description à proposer. En voici les plus saillants :

a) La forme nominalisée des Vsup.:

Nous avons observé que les Vsup. acceptent le changement de catégorie sans acquérir un emploi prédicatif et tout en gardant une fonction «support». Dans ce cas, la construction à Vsup. acquiert le statut de groupe nominal inclus dans une autre phrase:

Pazra: camalijjatan (faire accompli-opération= il a fait une opération);

Pizra:?u camalijjatin (forme nominalisée du verbe-opération= effectuer une opération);

Pintahal-hadi: $\theta u$ (se terminer accompli - art. déf.- conversation= la conversation se termina);

intiha:?ul-hadi: $\theta \mathrm{i}$ (forme nominalisée du verbe - art. déf.- conversation= la fin de la conversation).

La nominalisation des Vsup. en arabe est possible puisque, d'une manière générale, la nominalisation des formes verbales est quasi systématique. Ceci a pour cause le fait que le système dérivationnel arabe fonctionne par tableaux. Chaque tableau représente une matière consonantique à partir de laquelle sont dérivées les formes verbales et nominales selon des schèmes bien déterminés. Ainsi, pouvons-nous, à partir de la matière (3-r-j), former des dérivés tels que: zara:, ?azra: (verbes), zaraja:n, etc.

Nous constatons que ce type de séquences est très fréquent en arabe moderne ${ }^{5}$.

b) Les dérivés de la même racine et les relations paraphrastiques possibles:

C'est dans le même sens que nous évoquons les différents dérivés de la même racine que le Npred. Ainsi, si l'on prend le Npred «qara:r» (=décision) dans l'exemple suivant:

itta $\chi \alpha \delta a \mathrm{~N}_{0}$ qara:ran (bi...)

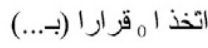

prendre la décision de...

nous constatons que, du point de vue morphologique, il est apparenté au verbe prédicatif «qarrara» (=décider) qui peut former la paraphrase: 
qarrara $\mathrm{N}_{0}($ (an...)

Nous nous contentons ici de remarquer que ce fait morphologique est quasi systématique en arabe. Il faudrait cependant préciser, par une étude des schémas d'arguments à générer, si tel ou tel verbe prédicatif (voire adjectif) constitue la paraphrase d'un emploi de Npred. En effet, cette systématicité morphologique ne reflète pas toujours une synonymie entre les phrases formées à partir d'un dérivé donné, comme nous le verrons dans les échantillons donnés en 3.

S'il est possible donc de parler de «racines prédicatives» pour la plupart des emplois, il faudra expliciter les informations concernant les cas contraires.

c) La construction en Npred $=\mathrm{N}_{0}$ :

C'est un cas très fréquent pour des raisons morphologiques, à savoir la multitude des schèmes verbaux pour une même racine (matière consonantique). Il a pour conséquence le fait que le "principe de co-référence ${ }^{6}$ » entre le sujet du V.sup et celui du N.préd n'est pas respecté. Examinons l'exemple suivant:

?azra:- $\mathrm{N}_{0}$-muћa:da $\theta \mathrm{a}:$ tin-maca- $\mathrm{N}_{1}$

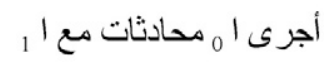

effectuer- $\mathrm{N}_{0}$-pourparlers-avec- $\mathrm{N}_{1}$

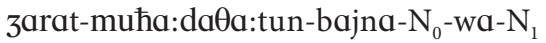

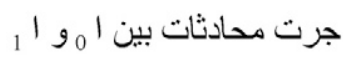

(se) dérouler-pourlarlers-entre- $\mathrm{N}_{0}$-et- $\mathrm{N}_{1}$

Entre les deux phrases, le passage du schème composé (?azra:) au schème simple est

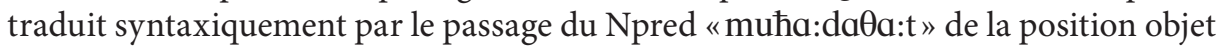
direct à la position sujet du Vsup. Plusieurs études ${ }^{7}$ ont montré que ce genre de construction fournit l'équivalent des tournures impersonnelles du français. Dans un grand nombre de cas, ce type de transformation avec des Vsup. d'occurrence permet de faire l'économie du sujet pour focaliser sur le procès lui-même.

\section{3. Échantillons de description de classes de Npred. en arabe}

Nous présentons dans ce qui suit quelques classes de Npred. extraites d'un corpus arabe moderne (journalistique, littéraire et lexicographique) (Ouerhani 2005).

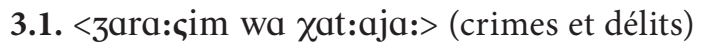

Cette classe se distingue par l'homogénéité sur le plan de l'actualisation par un groupe de verbes supports appropriés. En voici quelques exemples:

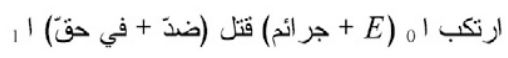

irtakaba- $\mathrm{N}_{0}-\left(E+\right.$ zara:Pim)-qatl-(didda + fi: haqqi) $-\mathrm{N}_{1}$ commettre- $\mathrm{N}_{0}-(E+$ crimes $($ de $))$-meurtre-contre- $\mathrm{N}_{1}$

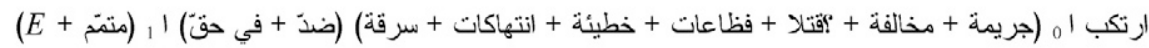

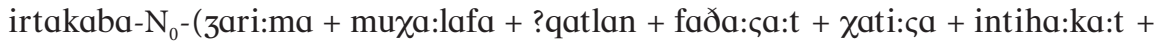
sariqatan)-(didda + fi: haqqi) $-\mathrm{N}_{1}$

commettre- $\mathrm{N}_{0}$ - (crime + transgression + meurtre + atrocités + péché + violations + vol)- contre- $\mathrm{N}_{1}$ 
Comme nous l'avons mentionné dans le paragraphe précédent, les verbes supports de cette classe portent déjà une valeur sémantique «minimale» négative héritée d'un emploi prédicatif lointain et inférée par le simple emploi de ces verbes. Voici la proposition de description de la classe:

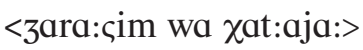

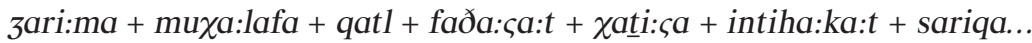

(crime + transgression + meurtre + atrocités + péché + violations + vol $)$

1- Nombre et nature des arguments:

$\mathrm{N}_{0}$ : hum $/ \mathrm{N}_{1}$ : hum-non hum/ $\mathrm{N}_{2}$ : didda, fi: haqqi

- Structures syntaxiques possibles ${ }^{9}$ :

Vsup- $\mathrm{N}_{0}$ - (quantifieur)- (classifieur)- dét: déf, indéf- <Npred>- (modifieur: adj., génitif)- prép.: didda, fi: haqqi- $\mathrm{N}_{1}$ - (loc.)- (tmps).

Vsup- (quantifieur)- (classifieur)- dét: déf, indéf.- $<\mathrm{Npred>}$ (modifieur: adj., génitif)prép.: didda, fi: haqqi- $\mathrm{N}_{1}$ - (loc.)- (tmps.) $\left.\left[\mathrm{N}_{0}=\mathrm{Npred}\right)\right]^{10}$

2- Verbes supports basiques:

qa:ma bi

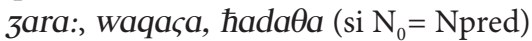

3- Déterminants:

- «nakira» (équivalent de l’indéfini)

- «maçrifa» (défini)

- Quantifieurs: cadad min (un certain nombre de), mazmu:ca min (un ensemble de), al-kaӨi:r min (beaucoup de)...

- Modifieurs: faði:ca (atroce), la: tuðtafar (impardonnable)

- Classifieurs: zari:ma, zara:?im

4- Verbes supports aspectuels:

Duratif: wa:sala

Inchoatif

Intensif

Itératif: karrara, zaddada, Paca:da, ৎa:wada

Ponctuel:

Progressif:

Terminatif

5- Verbes supports appropriés:

irtakaba, iztarama, Iqtarafa

6- Verbes supports métaphoriques

7- Restructurations:

a. Converses:

taçarrada $B$ li $<$ Npred $>\min ($ qibali) $A$

kana $B$ daћijjata <3ari:ma + faða:ca:t + intiha:ka:t + sariqa >min(qibali) $A$

b. Relations «paraphrastiques» avec le verbe morphologiquement apparenté:

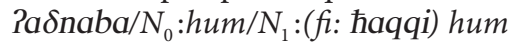

saraqa/ $N_{0}:$ hum $/ N_{1}:$ non hum $/ N_{2}$ : (min) hum

qatala $/ N_{0}:$ hum $/ N_{1}:$ hum, $<$ animaux $>,<$ végétaux $>$

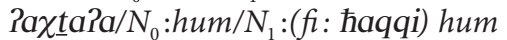

${ }^{*}$ intahaka/ $N_{0}:$ hum 
Pazrama/ $N_{0}:$ hum

iztarama $/ N_{0}:$ hum

Remarque: N1 est obligatoire dans tous ces exemples.

c. Passif:

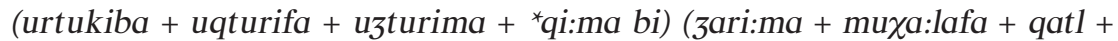

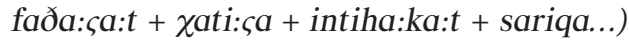

\section{2. <iztima:ৎa:t> (réunions)}

Ces noms prédicatifs appartiennent à la grande classe des événements organisés. En voici quelques exemples d'emploi:

$$
\text { عقدت اللجنة (اجتماعا + لقاء + ملتقى + ندوة + محادثات + * *فلا) (مك + زم + }
$$

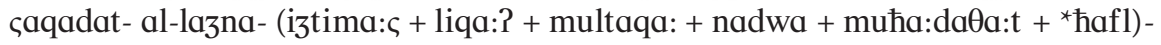
(loc. + tmps. $+E$ )

tenir- dét.- commission- (réunion / rencontre / congrès / colloque / pourparlers / ${ }^{\star}$ fête) (loc. + tmps. $+E)$

$$
\text { نظدت اللجنة (اجتماعا + لقاء + ملتقى + ندوة + محادثات + حفلا) (مك + زم + متمّم + Eمad) }
$$

naððamat- al-lazna- (iztima: $ৎ$ liqa:? + multaqa: + nadwa + muћa:daӨa:t + hafl)$($ loc. + tmps. $+E)$

organiser - (réunion / rencontre / congrès / colloque / pourparlers / fête) (loc. + tmps. $+E)$

$$
\text { انتظم (اجنماع + لقاء + ملتقى + ندوة + محادثات + حفل...) (مك + زم + منمّم + E) + (E) + }
$$

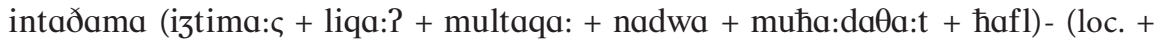
tmps. +E)

(s')organiser - (réunion / rencontre / congrès / colloque / pourparlers / fête) (loc. + tmps. +E)

$$
\text { (انعقد + التأم) (اجنماع + لقاء + ملنقى + ندوة + محادثات + * حفل ...) (مك + زم + منتمّ + (E) }
$$

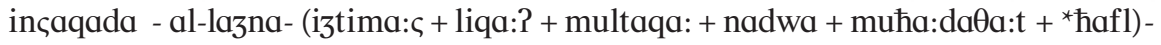
$($ loc. + tmps. + E)

tenir- dét.- commission- (réunion / rencontre / congrès / colloque / pourparlers / ^fête) $($ loc. + tmps. $+E)$

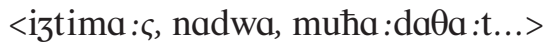

réunion, séminaire, pourparlers...

1- Nombre et nature des arguments:

$\mathrm{N}_{0}$ : hum/ $\mathrm{N}_{1}: /$

- Structures syntaxiques possibles:

Vsup- $\mathrm{N}_{0}$ - dét.: déf, indéf- (classifieur: 3awla min)- <Npred>- (modifieur: adj, génitif)- (loc)- (tmps)

Vsup- dét.: déf, indéf.- (classifieur: 3awla min) -<Npred>- (modifieur: adj, génitif)(loc)-(tmps) [Npred $=\mathrm{N}_{0}$ ]

2- Verbe(s) support(s) basiques:

3ara:, waqa $\varsigma$ a, $\left[\mathrm{N}_{0}=\right.$ Npred $]$ 
3- Déterminants:

- «nakira» (équivalent de l’indéfini)

- «macrifa» (défini)

- Quantifieurs: cadad min (un certain nombre de), mazmu:ৎa min (un ensemble de), al-kaӨi:r min (beaucoup de)...

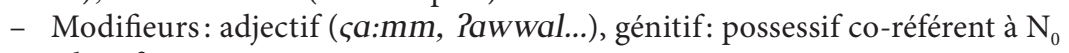

- Classifieurs:

4- Verbes supports aspectuels:

inchoatif: bada?a (fi:), Piftataћa, faraca (fi:),...

duratif: da:ma, wasala, tawa:şala, Pistamarra (fi:),...

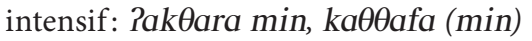

itératif: karrara, takarrara $\left[\mathrm{N}_{0}=\right.$ Npred $]$

ponctuel:

progressif:

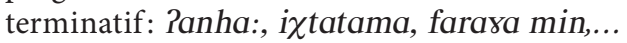

5- Verbes supports appropriés:

saqada

inçaqada, zara:, intaðama, ilta?ama [Npred $=\mathrm{N}_{0}$ ]

6- Verbes supports métaphoriques

7- Restructurations:

a. Converses

b. Relations «paraphrastiques» avec le verbe morphologiquement apparenté:

iztimaca/ $N_{0}:$ hum $/ N_{1}:(w a+$ bi)hum

Pintada: $/ N_{0}:$ hum $/ N_{1}:(w a+$ bi)hum

taћa:da $\theta a / N_{0}:$ hum $/ N_{1}:(w a+$ maca $)$ hum

c. Passif

Remarques concernant les relations paraphrastiques:

- Sur le plan morphologique, le verbe intada: existe bien dans le dictionnaire, mais nous n'avons pas trouvé d'emploi contemporain équivalent de la construction à verbe support, c'est-à-dire qu'il ne constitue pas la paraphrase de l'emploi du Npred. nadwa. Notons aussi qu'il existe un autre Npred. dérivé de la même matière consonantique (racine), à savoir muntada: qui appartient à la même classe et qui est actualisé, par ailleurs, par les Vsup. suivants:

(naððama + ?aqa:ma) $\mathrm{N}_{0}$ muntada:

$$
\text { (نظم + أقام) إم منتدى }
$$

(organiser + tenir) $\mathrm{N}_{0}$ forum/ rassemblement

organiser $\mathrm{N}_{0}$ un forum

Pour ce Npred. également, le verbe morphologiquement apparenté intada: ne forme pas une paraphrase.

- Notons que le «statut » grammatical de $\mathrm{N}_{1}$ n'est pas le même selon qu'il est introduit par la préposition wa d'un côté, ou les prépositions maça et bi de l'autre. En effet, dans le premier cas, il est présenté grammaticalement comme un deuxième agent ayant lui aussi le statut de «sujet» avec le $\mathrm{N}_{0}$, d'où l'appellation arabe de «mafçu:l maçahu» (littéralement «avec lui»). Tandis que dans le deuxième cas (introduction par maca et bi), il est grammaticalement complément, et la phrase focalise, de ce fait, sur $\mathrm{N}_{0}$ en tant qu'investigateur du procès. 
3.3. <çuquba> (punition/ sanction)

Voici quelques phrases qui illustrent l'emploi de cette classe de Npred.

$$
\text { (سلط + فرضَ) المكنب عقوبة على الفريق }
$$

(sallata + farada)-1-maktabu-cuqubatan-cala: -l-fari:qi

infliger/ adresser... accmpl- artdéf-bureau S-(une) punition/ sanction-prép sur-art. déf.-équipe

Le bureau a infligé une sanction à l'équipe)

(talaqqa: + $\chi$ adaça li) -N1-cuqu:ba:tin-min N0

recevoir accmpl- $\mathrm{N}_{1} S$ - des punitions/ sanctions- de/ de la part de- $\mathrm{N}_{0}$

$\mathrm{N}_{1}$ a subi des sanctions de $\mathrm{N}_{0}$

<cuqu:ba > punition/ sanction

1- Nombre et nature des arguments:

$\mathrm{N}_{0}$ : hum/ $\mathrm{N}_{1}$ : cala:, fi:-haqqi hum $/ \mathrm{N}_{1}$ :hum

- Structures syntaxiques possibles:

Vsup/ $\mathrm{N}_{0}$ / dét. : déf, indéf- <Npred $>$ (modifieur: adj, génitif)-(loc)- (tmps)

2- Verbes supports basiques

3- Déterminants:

- «nakira $»$ (équivalent de l'indéfini)

- «maçrifa» (défini)

- Quantifieurs

- Modifieurs: adjectifs (tels que: qa:sija «dure», Piqtisadijja, «économique»...), génitif: possessif co-référent à $\mathrm{N}_{0}, \ldots$

- Classifieurs

4- Verbes supports aspectuels:

Duratif: da:ma, tewa:sala, Pistamarra $\left[\mathrm{N}_{0}=\right.$ Npred]

Inchoatif: bada?a $\left[\mathrm{N}_{0}=\right.$ Npred $]$

Intensif

Itératif

Ponctuel

Progressif

Terminatif: Panha:

intaha: $\left[\mathrm{N}_{0}=\right.$ Npred $]$

5- Verbes supports appropriés:

sallata, farada, ictamada

6- Verbes supports métaphoriques

7- Restructurations:
a. Converses:

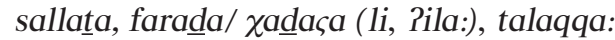
b. Relations "paraphrastiques» avec le verbe morphologiquement apparenté: §a:qaba $/ N_{0}:$ hum $/ N_{1}:$ hum
c. Passif:
furidat-cuqu:ba:tun- cala:-balad
Imposer passif accmpl- des punitions/ sanctions- prép sur- pays
Des sanctions ont été infligées à pays 
uctumidat-cuquba:tun-fi:-haqqi-balad-fi:-loc

Décider passif accmpl- des punitions/ sanctions- contre- pays- prép dans loc

Des sanctions ont été décidées contre pays à loc)

Remarque concernant le passif:

Elle concerne précisément le deuxième emploi où l'on trouve uniquement la forme passive du Vsup, alors que la forme active du même verbe n'est pas employée. Ajoutons, par ailleurs, qu'on trouve aussi l'adjectif apparenté à ce verbe associé aux mêmes noms prédicatifs en tant qu'épithète dans des groupes nominaux tels que: al-çuqu :ba:t al-muçtamada (les sanctions décidées).

Dans des cas pareils, il faudra intégrer cette information dans la grille de description: le fait que la forme passive uniquement d'un verbe est employée comme support. Ceci éviterait de générer, dans la traduction vers l'arabe, des constructions avec la forme active, alors qu'elle n'est pas employée.

Observons que ce type de construction est assez fréquent dans la presse arabe vu la valeur impersonnelle qu'elle véhicule. En effet, il permet de mettre l'accent sur le procès et la partie qui le subit, et d'omettre le sujet ou de le reléguer au second plan.

\section{Conclusion}

Nous avons essayé dans ce travail de montrer la nécessité de prendre en considération les faits spécifiques d'une langue donnée lors de l'élaboration des grilles de description linguistique en vue de la traduction automatique des Npred.

Pour une langue comme l'arabe, nous pensons que l'intégration de certains champs concernant notamment les relations entre des constructions dont les prédicats sont de formes morphologiques apparentées (dérivées de la même matière consonantique) est une condition nécessaire pour la confection de descriptions qui seraient en mesure de fournir toutes les informations nécessaires pour «comprendre», transférer et générer des constructions à Vsup. ou leurs équivalents.

Nous insistons sur le fait que ce ne sont là que des échantillons en vue de l'établissement d'un programme plus vaste pour l'élaboration de grilles de traitement pour la totalité des Npred. en arabe, et que ce type de description nécessite un travail sur un grand corpus. L'élaboration d'une méthodologie appropriée est en cours.

\section{NOTES}

1. Vu le grand nombre de ces travaux, nous nous contentons de renvoyer, entre autres, à la bibliographie du laboratoire disponible sur son site web.

2. Cf., par exemple, Alonso Ramos (1999, espagnol-français), Marques-Ranchhod (2000, portugaisfrançais) et Ouerhani (2000, arabe-français).

3. Cf. Gross (1989).

4. Rappelons que la classe des «humains» inclut les extensions par métonymie et par métaphore.

5. Ces remarques et conclusions ont été tirées de l'étude menée sur un grand corpus dans le cadre de notre thèse intitulée Les verbes supports en arabe moderne: étude syntactico-sémantique. Thèse de doctorat, Université de la Manouba (soutenue en juillet 2005).

6. Cf., entre autres, Harris (1964), Gross (1975), Vivès (1983).

7. Notamment, Badawi (1999), Girod et Larcher (2003), Ouerhani (2005).

8. Ce substantif joue le rôle de classifieur dans ce type de construction. Il est par ailleurs prédicatif dans des emplois du type: irtakaba zari:ma (commettre un crime). Cf. l'exemple suivant.

9. Elles indiquent les éléments de «la suite la plus longue» dans l'ordre de la réalisation de la phrase et mentionnent entre parenthèses des éléments facultatifs. 
10. Cette dernière précision (entre crochets) est en fait la raison d'existence de cette deuxième construction. Elle se caractérise par le fait que le Npred occupe la fonction de sujet grammatical et permet de ce fait d'omettre le sujet «réel». Elle est ainsi l'équivalent des constructions impersonnelles en français (cf. Ouerhani 2000).

\section{RÉFÉRENCES}

Alonso Ramos, M. (1998): Étude sémantico-syntaxique des constructions à verbe support, thèse de doctorat, Université de Monréal.

BADAWI, M. (1999): "A propos des aspects lexicaux du passif analytique en arabe moderne», Fachgruppe Sprachwissenschaft der Universität Konstanz, Arbeitspapier Nr. 98.

Blanco, X. et P.-A. Buvet (1999): «À propos de la traduction automatique des déterminants de l'espagnol et du français», META 44-4, pp. 526-545.

DANLOS, L. (1988): «Les problèmes posés par les verbes supports en traduction automatique», Actes du colloque LIANA.

DANLOS, L. (1994): «Coder des informations monolingues sur les noms pour éviter des règles bilingues sensibles au contexte», Langages 116. pp. 95-110.

Girod, A. et P. Larcher (2003): «Un phénomène de grammaticalisation en arabe moderne: le passif périphrastique», La grammaticalisation, la terminologie, Cercle Linguistique d'AixEn-Provence.

GiRy-SCHNeIDER, J. (1987): Les prédicats nominaux en français: Les phrases simples à verbes supports, Droz, Genève-Paris.

Gross, G. (1989): Les constructions converses du français, Genève, Droz.

Gross, G. (1992): «Forme d'un dictionnaire électronique», Clas, A. et H. Safar (dir.), L'environnement traductionnel, Syllery, Presses de l'Université du Québec.

Gross, G. (1995): «Une sémantique nouvelle pour la traduction automatique: les classes d'objets», La Tribune des Industries de la Langue et de l'Information électronique, Paris.

Gross, G. (1996): «Prédicats nominaux et compatibilité aspectuelle», Langages 121, pp. 54-72.

Gross, M. (1981): «Les bases empiriques de la notion de prédicat sémantique», Langages 63, Formes syntaxiques et prédicats sémantiques", pp. 7-52.

Gross, M. (1998): «La fonction sémantique des verbes supports», Travaux de Linguistique 37.

HARris, Z.-S. (1976): Notes du cours de syntaxe, Paris, Seuil.

MejRI, S. (1993): «Séquences figées et expression de l'intensité. Essai de description sémantique», Cahiers de lexicologie 65, pp. 111-122.

Mejri, S. (1997): Le figement lexical, Thèse d'Etat, Tunis, Publications de la Faculté de Lettres de la Manouba.

MeL'ČUK, I. (2003) : «Collocations: définition, rôle et utilité», Les collocations: analyse et traitement; Travaux et recherches en linguistique appliquée, Série E- № 1, Amsterdam, pp 2331.

Ouerhani, B. (2000): «La problématique de la traduction des verbes supports, de l'arabe vers le français", Actes du Colloque Traduction Humaine, Traduction Automatique, Interprétation, Tunis 28-29-30 septembre 2000, Volume 1, pp 133- 148.

Ouerhani, B. (2003): «Verbes supports: polysémie et polylexicalité», Syntaxe et Sémantique 5, Presses Universitaires de Caen.

OUERHANI, B. (2005): Les verbes supports en arabe moderne: étude syntactico-sémantique, thèse de doctorat (en arabe), Université de la Manouba.

Ouerhani, B. (à paraître): «Les critères de définition des verbes supports, entre le français et l'arabe», Syntaxe et Sémantique, Presses Universitaires de Caen.

RANCHHOD-MARques, E. (2000): «Problème de traduction automatique des constructions à verbes supports», Linguisticae investigationes 23, pp. 253-267.

VIVÈs, R. (1983): Avoir, prendre, perdre: constructions à verbes supports et extensions aspectuelles, Thèse de doctorat, Université Paris VIII. 